\title{
On the equivalence between global recurrence and the existence of a smooth Lyapunov function for hybrid systems
}

\author{
Anantharaman Subbaraman Andrew R. Teel
}

\begin{abstract}
We study a weak stability property called recurrence for a class of hybrid systems. An open set is recurrent if there are no finite escape times and every complete trajectory eventually reaches the set. Under sufficient regularity properties for the hybrid system we establish that the existence of a smooth, radially unbounded Lyapunov function that decreases along solutions outside an open, bounded set is a necessary and sufficient condition for recurrence of that set. Recurrence of open, bounded sets is robust to sufficiently small state dependent perturbations and this robustness property is crucial for establishing the existence of a Lyapunov function that is smooth. We also highlight some connections between recurrence and other well studied properties like asymptotic stability and ultimate boundedness.
\end{abstract}

\section{INTRODUCTION}

Hybrid systems are a class of dynamical systems that combine continuous-time evolution and discrete-time events. Several frameworks have been proposed in the literature for analysis of hybrid systems. We refer the reader to [1], [2] and [3] for details. Converse Lyapunov theorems are used to establish the equivalence between asymptotic stability properties and the existence of Lyapunov-like functions that satisfy certain decrease conditions along solutions. Applications of converse theorems in stabilization and robust stability analysis can be found in [4], [5] and [6]. For continuous-time systems converse theorems related to asymptotic stability are established in [6], [7] and [8]. See [9], [10] and [11] for similar results in the discrete-time case. Converse theorems for asymptotic stability of compact sets for a class of hybrid systems has been established in [12] and [13]. In this paper, we establish a converse theorem for a similar class of hybrid systems considered in [12] but for a weaker property called recurrence.

Recurrence of an open, bounded set is a weak stability property that is frequently studied in the literature for stochastic systems. We refer the reader to [14], [15] and [16] for details. Loosely speaking, recurrence of an open, bounded set implies that solutions visit the set infinitely often with probability one. It is a weaker notion of stability compared to probabilistic notions of asymptotic stability but nevertheless useful in many applications. In particular, for the problem of RF-source seeking using Micro Aerial Vehicles (MAV) considered in [17] and [18], it is shown

\footnotetext{
The authors are with the Electrical and Computer Engineering Department, University of California, Santa Barbara, CA 93106-9560.anantharman@umail.ucsb.edu, teel@ece.ucsb.edu. Research supported in part by AFOSR FA9550-12-1-0127 and NSF ECCS-1232035.
} 
that asymptotic properties like convergence are difficult to establish in the presence of persistent disturbances and recurrence proves to be a useful alternative.

In this paper we study the recurrence property not for stochastic systems, but for a class of non-stochastic hybrid systems. To the best of the authors' knowledge this property has not been studied in detail for non-stochastic hybrid systems. Although recurrence is a weak property in the context of stochastic systems, we show that recurrence of an open, bounded set is actually equivalent to establishing uniform ultimate boundedness of solutions for non-stochastic systems. A similar observation is made for discrete-time deterministic systems in [15], although it is noted that such an equivalence does not hold true for recurrence in stochastic systems. The importance of ultimate boundedness in control design for uncertain systems is explained in [19] and [20]. We also note that recurrence-like properties are studied for deterministic systems in [21, Chapter 7] and [22, Chapter 1].

Necessary and sufficient conditions for global recurrence in terms of Lyapunov functions are established in this work. For discrete-time stochastic systems with non-unique solutions, i.e., stochastic difference inclusions, the results in [23] establish the equivalence between recurrence of an open, bounded set and the existence of a smooth Lyapunov function that decreases on average outside the recurrent set. This is achieved by establishing robustness of recurrence to various state dependent perturbations. We follow a similar approach in this paper to establish a converse Lyapunov theorem, but for a class of non-stochastic hybrid systems. A converse theorem for a stronger version of recurrence called positive recurrence is established for discrete-time stochastic systems in [15] and in [14, Thm 3.26] for a class of switching diffusion processes.

Converse theorems for recurrence in discrete-time deterministic systems is developed in [15, Thm 11.2.1] although the Lyapunov function generated is merely upper semicontinuous. Using the robustness of recurrence to various state dependent perturbations we construct a smooth Lyapunov function for the converse theorem. Robustness of recurrence to sufficiently small perturbations is due to the hybrid system satisfying good regularity properties. Without the regularity properties, it is not guaranteed that recurrence or other stability properties are robust. We exploit the robustness to go from a preliminary non-smooth Lyapunov function to a smooth Lyapunov function for recurrence by utilizing the construction in [12]. We also make connections of this property to asymptotic stability and ultimate boundedness. In [24] a robust boundedness problem is studied for continuous-time systems that uses the notion of first hitting times to certain forward invariant compact sets. In this paper, we will use similar tools, but to study the recurrence property. Finally, recurrence for non-stochastic systems has extra consequences compared to the stochastic counterpart. This paper will also briefly highlight some of these differences.

The rest of the paper is organized as follows. Section II presents the basic notation and definitions to be used in the paper. Section III introduces the hybrid systems framework that will be considered in the rest of the paper. Section IV presents the definition of recurrence and its uniform version. Section V makes connections between recurrence and other well studied properties. Section VI presents the main results. Section VII presents an equivalent characterization of recurrence which will be used to prove the main results of the paper. The proof of the converse theorem is presented in Section VII. Section IX presents some concluding comments and future work. 


\section{NOTATION AND BASIC DEFINITION}

For a closed set $S \subset \mathbb{R}^{n}$ and $x \in \mathbb{R}^{n},|x|_{S}:=\inf _{y \in S}|x-y|$ is the Euclidean distance of $x$ to $S$. Let $\mathbb{B}, \mathbb{B}^{o}$ denote the closed and open unit ball in $\mathbb{R}^{n}$. Given a closed set $S \subset \mathbb{R}^{n}$ and $\epsilon>0, S+\epsilon \mathbb{B}$ represents the set $\left\{x \in \mathbb{R}^{n}:|x|_{S} \leq \epsilon\right\} . \mathbb{R}_{\geq 0}$ denotes the non-negative real numbers; $\mathbb{Z}_{\geq 0}$ denotes the non-negative integers. For $c \geq 0$ and a function $V: \mathbb{R}^{n} \rightarrow \mathbb{R}_{\geq 0}, L_{V}(c):=\left\{x \in \mathbb{R}^{n}: V(x)=c\right\}$. A set-valued mapping $M: \mathbb{R}^{p} \rightrightarrows \mathbb{R}^{n}$ is outer semicontinuous if, for each $\left(x_{i}, y_{i}\right) \rightarrow(x, y) \in \mathbb{R}^{p} \times \mathbb{R}^{n}$ satisfying $y_{i} \in M\left(x_{i}\right)$ for all $i \in \mathbb{Z}_{\geq 0}, y \in M(x)$. A mapping $M$ is locally bounded if, for each bounded set $K \subset \mathbb{R}^{p}, M(K):=\bigcup_{x \in K} M(x)$ is bounded. A function $\Psi: \mathbb{R}^{n} \rightarrow \mathbb{R}_{\geq 0}$ is upper semicontinuous if for every sequence $\left\{x_{i}\right\}_{i=0}^{\infty}$ such that $x_{i} \rightarrow x$, we have $\lim \sup _{i \rightarrow \infty} \Psi\left(x_{i}\right) \leq \Psi(x)$. For $S \subset \mathbb{R}^{n}$, the symbol $\mathbb{I}_{S}$ denotes the indicator function of $S$ i.e., $\mathbb{I}_{S}(x)=1$ for $x \in S$ and $\mathbb{I}_{S}(x)=0$ otherwise. For vectors $f_{1}, f_{2} \in \mathbb{R}^{n},\left\langle f_{1}, f_{2}\right\rangle:=f_{1}^{T} f_{2}$ denotes the inner product.

\section{PReliminaries ON HYBRID SYSTEMS}

We follow the mathematical framework in [1] for modeling hybrid systems. As explained in [25, Chapter 1] other models for describing hybrid systems can be encompassed within the framework of [1]. So we consider a class of stochastic hybrid systems considered in [1] with a state $x \in \mathbb{R}^{n}$ written formally as

$$
\begin{aligned}
& \dot{x} \in F(x), \quad x \in C \\
& x^{+} \in G(x), \quad x \in D .
\end{aligned}
$$

where $C, D \subset \mathbb{R}^{n}$ represent the flow and jump sets (where continuous and discrete evolution of the state is permitted) respectively and $F, G$ represent the flow and jump maps respectively. In essence, the continuous-time dynamics is modeled by a differential inclusion and the discrete-time dynamics is modeled by a difference inclusion. We consider a very general class of hybrid systems modeled by set-valued mappings as opposed to single-valued mappings as set-valued mapping arise naturally in the context of robustness analysis and study of ISS properties of hybrid systems with inputs. We refer the reader to [12], [25] and [1] for more details.

The solution concept for systems of the form (1) is explained in detail in [25, Chapter 2]. We define solutions on a generalized time domain that uses two variables $t, j$ to keep track of the continuous evolution of the state and the number of jumps elapsed. To define solutions to (1) we require the notion of a hybrid time domain: a subset $E$ of $\left(\mathbb{R}_{\geq 0} \times \mathbb{Z}_{\geq 0}\right)$, which is the union of infinitely many intervals of the form $\left[t_{j}, t_{j+1}\right] \times\{j\}$, where $0=t_{0} \leq t_{1} \leq t_{2} \leq \ldots$, or finitely many of such intervals, with the last one possibly of the form $\left[t_{j}, t_{j+1}\right] \times\{j\}$, $\left[t_{j}, t_{j+1}\right) \times\{j\}$, or $\left[t_{j}, \infty\right) \times\{j\}$. A function $\phi: E \rightarrow \mathbb{R}^{n}$ that maps a hybrid time domain to the Euclidean space and for which $t \mapsto \phi(t, j)$ is locally absolutely continuous for fixed $j$ is called a hybrid arc.

A hybrid arc is a solution to (1) if $\phi(0,0) \in C \cup D$ and:

1) for all $j \in \mathbb{Z}_{\geq 0}$ and almost all $t$ such that $(t, j) \in \operatorname{dom} \phi: \phi(t, j) \in C, \dot{\phi}(t, j) \in F(\phi(t, j))$

2) for all $(t, j) \in \operatorname{dom} \phi$ such that $(t, j+1) \in \operatorname{dom} \phi: \phi(t, j) \in D, \phi(t, j+1) \in G(\phi(t, j))$. 
A solution to the hybrid system is called maximal if it cannot be extended, and complete if its domain is unbounded. We will represent the hybrid system through its data as

$$
\mathcal{H}:=(C, F, D, G)
$$

We denote by $\mathcal{S}_{\mathcal{H}}(K)$ the set of all maximal solutions starting from the set $K \subset \mathbb{R}^{n}$ for the hybrid system $\mathcal{H}$. We assume throughout the paper that $\mathcal{H}$ satisfies certain regularity properties listed below.

Standing Assumption 1. The data $\mathcal{H}$ of the hybrid system satisfies the following conditions:

1) The sets $C, D \subset \mathbb{R}^{n}$ are closed.

2) The mapping $F$ is outer semicontinuous, locally bounded, convex valued and non-empty on $C$.

3) The mapping $G$ is outer semicontinuous, locally bounded and non-empty on $D$.

If $F, G$ are single-valued mappings, then Standing Assumption 1 reduces to the mappings $f, g$ being continuous on $C$ and $D$ respectively. The main motivation for such assumptions is to ensure that stability properties are robust for the hybrid system. The robustness of asymptotic stability to sufficiently small state dependent perturbations under the conditions of Standing Assumption 1 is established in [12]. The system (1a) is said to have no finite escape times if there are no solutions of (1a) that escape to infinity at a finite time. In the rest of this paper, we will establish similar equivalences for the weaker property of recurrence and also illustrate using examples cases where such equivalences can fail due to the conditions of Standing Assumption 1 not being satisfied.

\section{RECURRENCE AND UNIFORM RECURRENCE}

In this section we define the notion of recurrence for sets.

Definition 1. A set $\mathcal{O} \subset \mathbb{R}^{n}$ is said to be globally recurrent for the hybrid system $\mathcal{H}$ in (2) if there are no finite escape times for (1a) and for each complete solution $\phi \in \mathcal{S}_{\mathcal{H}}(C \cup D)$, there exists $(t, j) \in$ dom such that $\phi(t, j) \in \mathcal{O}$.

Loosely speaking, this means that from every initial condition, solutions either stop or hit the set $\mathcal{O}$ and solutions do not exhibit finite escape times. The recurrence definition does not impose any invariance-like property for the set $\mathcal{O}$. Hence, solutions that start from the set $\mathcal{O}$ can leave the set. Recurrence of the set $\mathcal{O}$ also does not impose any stability-like conditions since solutions that start close to the set $\mathcal{O}$ need not stay close. In this respect, recurrence is different from the frequently studied asymptotic stability property. Nevertheless, there are some connections between recurrence and properties like ultimate boundedness and asymptotic stability. Such aspects are explored in Section $\mathrm{V}$. Another consequence of the recurrence property is that for complete solutions, recurrence for the set $\mathcal{O}$ implies that solutions have to visit the set $\mathcal{O}$ infinitely often.

Definition 2. A set $\mathcal{O} \subset \mathbb{R}^{n}$ is said to be uniformly globally recurrent for $\mathcal{H}$ in (2) if there are no finite escape times for (1a) and for each compact set $K$, there exists $T>0$ such that for each solution $\phi \in \mathcal{S}_{\mathcal{H}}(K)$, either $t+j<T$ for all $(t, j) \in$ dom $\phi$ or there exists $(t, j) \in$ dom $\phi$ such that $t+j \leq T$ and $\phi(t, j) \in \mathcal{O}$. 
The following example illustrates that the recurrence property is useful in the context of systems affected by persistent disturbances.

Example 1: Consider a discrete-time system with state $(x, d, \ell)$, parameters $\rho \in(0,1), M>0$ and the following system dynamics:

$$
\begin{aligned}
& x^{+}=d \\
& d^{+} \in\{0,1\} \\
& \ell^{+}=d(\ell+1-\rho)+(1-d) \max \{0, \ell-\rho\} .
\end{aligned}
$$

The dynamics is restricted to the set $D=\mathbb{R} \times\{0,1\} \times[0, M]$. The state $\ell$ basically captures a constraint on how often the disturbance $d$ can take the value $d=1$. As noted in [26], if the disturbance $d=1$ for all time steps, then the state $\ell$ grows out of the bound $[0, M]$ and solutions stop. Complete solutions for the system represented by $j \mapsto(x(j), d(j), \ell(j))$ satisfy $\sum_{j=j_{1}}^{j_{2}-1} d(j) \leq M+\rho\left(j_{2}-j_{1}\right)$ for integers $j_{1} \leq j_{2}$. See [26, Lemma 3] for more details. It can be easily observed that the set $\mathcal{A}=[0,1] \times\{0,1\} \times[0, M]$ is smallest possible globally asymptotically stable set. It follows from [26, Remark 2] that whenever $j_{2}-j_{1} \geq(M+1) /(1-\rho)$, there exists $j \in\left\{j_{1}, \ldots, j_{2}-1\right\}$ such that $d(j)=0$. Hence for complete solutions $(x(j), d(j), \ell(j))$, whenever $j_{2}-j_{1} \geq(M+1) /(1-\rho)$, there exists $j \in\left\{j_{1}, \ldots, j_{2}\right\}$ such that $x(j)=0$. It now follows that the set $\mathcal{O}=\varepsilon \mathbb{B} \times\{0,1\} \times[0, M]$ is globally recurrent for every $\varepsilon \geq 0$. Hence, the recurrence property provides a sharper characterization of the behavior of the solutions in the presence of a persistent disturbance $d$ as the set $\mathcal{O}$ is smaller than $\mathcal{A}$. We observe that any open set containing $\mathcal{O}$ is also globally recurrent. We note that the applications in [17] and [18] encounter similar persistent disturbances in the context of source seeking and recurrence is studied since other asymptotic convergence properties are not suitable to describe the solution behavior.

Recurrence is a property that is studied with respect to open sets for a variety of reasons. Firstly, we consider open, bounded sets to establish robustness of the recurrence property. Secondly, equivalence between uniform and non-uniform versions of recurrence hold only for open sets. These aspects will be illustrated through examples in the sections that follow.

The following result establishes equivalence between uniform and non-uniform recurrence when $\mathcal{O}$ is open and bounded under mild regularity properties for $\mathcal{H}$ stated in Standing Assumption 1. We provide a proof for completeness although this result has been established for a wider class of stochastic hybrid systems in [27, Thm 6] (or equivalently in [28, Prop 3.1]).

Proposition 1. An open, bounded set $\mathcal{O}$ is globally recurrent for $\mathcal{H}$ in (2) if and only if it is uniformly globally recurrent for $\mathcal{H}$.

Proof. $\Leftarrow$ Follows immediately from the definitions.

$\Rightarrow$ Suppose $\mathcal{O}$ is not uniformly globally recurrent. Then, there exists a compact set $K$ such that for every $i \in \mathbb{Z}_{>0}$, there exists a solution $\phi_{i} \in \mathcal{S}_{\mathcal{H}}(K)$ such that there exists $(t, j) \in \operatorname{dom} \phi_{i}$ satisfying $t+j>i$ and for all $(t, j) \in \operatorname{dom}\left(\phi_{i}\right)$ satisfying $t+j \leq i, \phi_{i}(t, j) \notin \mathcal{O}$. Due to compactness of $K$, and absence of finite escape times, it 
follows from [25, Prop 6.13] that the sequence of solutions $\phi_{i}$ is locally eventually bounded ${ }^{1}$. Then, [29, Thm 4.4] states that the sequence $\phi_{i}$ admits a converging subsequence $\psi_{i}$ that converges to a complete solution $\psi \in \mathcal{S}_{\mathcal{H}}(K)$. From recurrence of $\mathcal{O}$, there exists $(t, j)$ such that $\psi(t, j) \in \mathcal{O}$. From the definition of convergence of hybrid arcs, there exists a sequence $\left\{t_{i}, j_{i}, \psi_{i}\left(t_{i}, j_{i}\right)\right\}$ such that $t_{i} \rightarrow t, j_{i} \rightarrow j$ and $\psi_{i}\left(t_{i}, j_{i}\right) \rightarrow \psi(t, j)$. Since $\mathcal{O}$ is open, for $i$ large enough $\psi_{i}\left(t_{i}, j_{i}\right) \in \mathcal{O}$. This contradicts the initial assumption and establishes uniform recurrence of $\mathcal{O}$.

Without Standing Assumption 1, Proposition 1 is not necessarily true and the following example illustrates it.

Example 2: Consider $\mathcal{H}=(\varnothing, \varnothing, \mathbb{R}, g)$ where $g(x)=(\max \{0, x\})^{2}$ if $x<1$ and $g(x)=0$ otherwise.

Consider an open neighborhood of the origin of the form $\mathcal{O}:=(-\varepsilon, \varepsilon)$ for any $\varepsilon \in(0,1)$. Then for every initial condition $x \in \mathbb{R}^{n}$, all solutions reach the set $\mathcal{O}$ and hence $\mathcal{O}$ is globally recurrent. Now for every compact set $K \subset \mathbb{R}$ such that $\{1\} \in \operatorname{int}(K)$, the solutions that start arbitrarily close to the left of $x=1$ takes arbitrarily long times to reach the set $\mathcal{O}$ and hence the set $\mathcal{O}$ is not uniformly globally recurrent. The mapping $g$ is discontinuous at the point $x=1$ and hence does not satisfy Standing Assumption 1. Examples that illustrate this issue for stochastic hybrid systems are in [16].

The next example illustrates how Proposition 1 can fail if the set $\mathcal{O}$ is not open. The equivalence with uniform recurrence is crucial to the development of the converse Lyapunov theorem established later in the paper.

Example 3: Consider a continuous-time system with $C=\mathbb{R}^{2}$ and the following dynamics

$$
\dot{x}_{1}=x_{2}-x_{1}\left(x_{1}^{2}+x_{2}^{2}-1\right), \quad \dot{x}_{2}=-x_{1}-x_{2}\left(x_{1}^{2}+x_{2}^{2}-1\right) .
$$

Let $\epsilon \in(0,1)$. It can be observed that the closed set $\mathcal{O}:=\{0\} \cup\{x:|x| \in[1-\epsilon, 1+\epsilon]\}$ is globally recurrent. Moreover, for solutions starting closer to the origin, it takes arbitrarily long time to reach the set $\mathcal{O}$. Hence, uniform recurrence fails even though the system satisfies the conditions of Standing Assumption 1.

\section{RECURRENCE AND OTHER PROPERTIES}

In this section we make connections between recurrence and other properties like ultimate boundedness and asymptotic stability.

\section{A. Connection to Ultimate boundedness}

Definition 3. The solutions of $\mathcal{H}$ in (2) are uniformly ultimately bounded if there are no finite escape times and there exists $M>0$ such that for each $\Delta>0$ there exists $T>0$ such that for every $\phi \in \mathcal{S}_{\mathcal{H}}(\Delta \mathbb{B})$ either $t+j<T$ for all $(t, j) \in$ dom $\phi$ or $\phi(t, j) \in M \mathbb{B}$ for all $(t, j) \in$ dom $\phi$ satisfying $t+j \geq T$.

Proposition 2. The solutions of $\mathcal{H}$ in (2) are uniformly ultimately bounded if and only if there exists an open, bounded set $\mathcal{O}$ that is globally recurrent for $\mathcal{H}$.

\footnotetext{
${ }^{1} \mathrm{~A}$ sequence of solutions $\phi_{i}$ is called locally eventually bounded if for every $\tau \geq 0$, there exists $i^{*}$ and $M>0$ such that for all $i \geq i^{*}$ and all $(t, j) \in \operatorname{dom}\left(\phi_{i}\right)$ with $t+j \leq \tau, \phi_{i}(t, j) \in M \mathbb{B}$. We refer the reader to [1] and [25, Definition 5.24] for more details.
} 
Proof. It follows from the definition that ultimate boundedness of solutions of $\mathcal{H}$ implies that the set $\mathcal{O}=(M+1) \mathbb{B}^{o}$ is globally recurrent for $\mathcal{H}$.

Next, we establish that recurrence of an open, bounded set $\mathcal{O}$ for $\mathcal{H}$ implies uniform ultimate boundedness of solutions of $\mathcal{H}$. We first claim that the reachable ${ }^{2}$ set (in infinite hybrid time) from the compact set $\overline{\mathcal{O}}$ is bounded. It follows from [25, Prop 6.13] that there exists a compact set $K_{1}$ such that $\mathcal{R}_{\leq 2}(\overline{\mathcal{O}}) \subset K_{1}$. Let $T>0$ be such that the condition of uniform recurrence holds from the set $K_{1}$. Then, using [25, Prop 6.13], it follows that there exists a set $K_{2}$ such that $\mathcal{R}_{\leq T}\left(K_{1}\right) \subset K_{2}$. Then, we claim that $\cup_{\tau \geq 0} \mathcal{R}_{\tau}(\overline{\mathcal{O}}) \subset K_{2}$. Let $\phi \in \mathcal{S}_{\mathcal{H}}(\overline{\mathcal{O}})$. If $t+j \leq T+2$ for all $(t, j) \in \operatorname{dom} \phi$ then $\phi(t, j) \in K_{2}$ for all $(t, j) \in \operatorname{dom} \phi$. If not, there exists $\left(t_{1}, j_{1}\right)$ such that $t_{1}+j_{1} \in[1,2]$ and $\phi(s, i) \in K_{1} \subset K_{2}$ for $s+i \leq t_{1}+j_{1}$ and $\phi\left(t_{1}, j_{1}\right) \in \mathcal{O}$ or $\phi\left(t_{1}, j_{1}\right) \in K_{1} \backslash \mathcal{O}$. For the second case, there exists $\left(t_{2}, j_{2}\right)$ such that $0 \leq t_{2}+j_{2}-\left(t_{1}+j_{1}\right) \leq T$ such that $\phi\left(t_{2}, j_{2}\right) \in \mathcal{O}$ and $\phi(s, i) \in K_{2}$ for $s+i \leq t_{2}+j_{2}$. We now iterate the same argument to prove that the reachable set from $\overline{\mathcal{O}}$ is bounded. Next, we choose $M>0$ sufficiently large so that $K_{2} \subset M \mathbb{B}$. Now let $\Delta>0$. From the definition of uniform global recurrence there exists $T>0$ such that for each solution $\phi \in \mathcal{S}_{\mathcal{H}}(\Delta \mathbb{B})$, either $t+j<T$ for all $(t, j) \in \operatorname{dom} \phi$ or there exists $(t, j) \in \operatorname{dom} \phi$ such that $t+j \leq T$ and $\phi(t, j) \in \mathcal{O}$. Then, from $\mathcal{O}$ being recurrent, and the reachable set from $\mathcal{O}$ being bounded, it follows that there are no finite escape times and for every $\phi \in \mathcal{S}_{\mathcal{H}}(\Delta \mathbb{B})$ either $t+j<T$ for all $(t, j) \in \operatorname{dom} \phi$ or $\phi(t, j) \in M \mathbb{B}$ for all $(t, j) \in \operatorname{dom} \phi$ satisfying $t+j \geq T$. This establishes uniform ultimate boundedness.

\section{B. Connection to asymptotic stability}

Asymptotic stability is a widely studied property for dynamical systems.

Definition 4. A closed set $\mathcal{A}$ is uniformly globally stable (UGS) for $\mathcal{H}$, if there exists a class- $\mathcal{K}_{\infty}$ function $\alpha$ such that for every solution $\phi$ to $\mathcal{H},|\phi(t, j)|_{\mathcal{A}} \leq \alpha\left(|\phi(0,0)|_{\mathcal{A}}\right)$ for every $(t, j) \in \operatorname{dom}(\phi)$.

Definition 5. A closed set $\mathcal{A}$ is uniformly globally attractive for $\mathcal{H}$, if there are no finite escape times for (1a) and for every $\varepsilon>0, r>0$ there exists a $T>0$ such that for every solution $\phi$ to $\mathcal{H}$ with $|\phi(0,0)|_{\mathcal{A}} \leq r,(t, j) \in \operatorname{dom}(\phi)$ and $t+j \geq T$ imply $|\phi(t, j)|_{\mathcal{A}} \leq \varepsilon$.

A closed set $\mathcal{A}$ is uniformly globally asymptotically stable (UGAS) for $\mathcal{H}$ it is uniformly globally stable and uniformly globally attractive for $\mathcal{H}$. In [28], it is established that UGAS of a closed set can be expressed in terms of UGS and uniform global recurrence of open neighborhoods of the closed set. The following result is proved in [28, Prop 2.2] for a wide class of stochastic hybrid systems, so we only state the result here.

Proposition 3. If the closed set $\mathcal{A} \subset \mathbb{R}^{n}$ is UGS for $\mathcal{H}$ and, for every $\varepsilon>0$, the open set $\mathcal{A}+\varepsilon \mathbb{B}^{\circ}$ is uniformly globally recurrent for $\mathcal{H}$, then the set $\mathcal{A}$ is UGAS for $\mathcal{H}$.

In fact, the existence of an open, bounded recurrent set $\mathcal{O}$ for $\mathcal{H}$ implies that there exists a compact set $\mathcal{A}$, that

\footnotetext{
${ }^{2}$ The reachable set from a set $S$ within hybrid time $\tau$ is defined as $\mathcal{R}_{\leq \tau}(S):=\{\phi(t, j): \phi(0,0) \in S$ and $t+j \leq \tau\}$. The reachable set from a set $S$ in infinite hybrid time is $\cup_{\tau \geq 0} \mathcal{R}_{\tau}(S)$.
} 
is UGAS for $\mathcal{H}$. This is a consequence of the reachable set from $\mathcal{O}$ being bounded and [25, Corollary 7.7]. The proof of the following result is presented in the appendix.

Proposition 4. Let the open, bounded set $\mathcal{O} \subset \mathbb{R}^{n}$ be globally recurrent for $\mathcal{H}$ in (2). Then, there exists a compact set $\mathcal{A}$ that is UGAS for $\mathcal{H}$.

\section{Weak sufficient conditions for recurrence}

We will briefly state some weak sufficient conditions for recurrence in terms of non-strict Lyapunov functions. Such conditions for asymptotic stability are established in [25]. The next result is stated in [30] for a wide class of stochastic hybrid systems.

A continuous function $\widehat{V}: \mathbb{R}^{n} \rightarrow \mathbb{R}_{\geq 0}$ is a weak-Lyapunov function relative to a open, bounded set $\mathcal{O} \subset \mathbb{R}^{n}$ for the system $(C, F, D, G, \mu)$ if $\widehat{V}$ is radially unbounded and satisfies

$$
\begin{aligned}
\widehat{V}(\phi(t)) & \leq \widehat{V}\left(x_{0}\right), \forall x_{0} \in C \cap\left(\mathbb{R}^{n} \backslash \mathcal{O}\right), t \in \operatorname{dom}(\phi), \phi \in \mathcal{S}_{C \cap\left(\mathbb{R}^{n} \backslash \mathcal{O}\right)}^{F}\left(x_{0}\right) \\
\max _{g \in G\left(x_{0}\right) \cap\left(\mathbb{R}^{n} \backslash \mathcal{O}\right)} \widehat{V}(g) & \leq \widehat{V}\left(x_{0}\right), \forall x_{0} \in D \cap\left(\mathbb{R}^{n} \backslash \mathcal{O}\right)
\end{aligned}
$$

where $\mathcal{S}_{C \cap\left(\mathbb{R}^{n} \backslash \mathcal{O}\right)}^{F}\left(x_{0}\right)$ refers to solutions of the constrained system $\dot{x} \in F(x), x \in C \cap\left(\mathbb{R}^{n} \backslash \mathcal{O}\right)$ from initial condition $x_{0}$.

Theorem 1. Let $\widehat{V}$ be a weak-Lyapunov function relative to a open, bounded set $\mathcal{O} \subset \mathbb{R}^{n}$ for the system $\mathcal{H}$. Then, $\mathcal{O}$ is globally recurrent if and only if for every $c \geq 0$ for which $L_{V}(c) \cap\left(\mathbb{R}^{n} \backslash \mathcal{O}\right)$ is non-empty there does not exist a complete solution $\phi$ that remains in the set $L_{V}(c) \cap\left(\mathbb{R}^{n} \backslash \mathcal{O}\right)$.

\section{ROBUST GLOBAL RECURRENCE AND A CONVERSE LYAPUNOV THEOREM}

In this section we present results on robustness of recurrence to sufficiently small state dependent perturbations and also describe a Lyapunov function based characterization for recurrence. We also illustrate using examples the importance of Standing Assumption 1 in issues relating to robustness and the existence of smooth Lyapunov functions.

\section{A. Robustness of recurrence to state dependent inflations}

We now establish a series of robustness results that will eventually be applied to the development of converse Lyapunov theorems in a similar manner as in [23]. Firstly, since we do not insist on solutions to $\mathcal{H}$ being complete we analyze an inflated system of $\mathcal{H}$ for which maximal solutions are complete and for which recurrence properties are preserved. This inflated system is later used in the construction of a preliminary non-smooth Lyapunov function to certify recurrence of an open, bounded set for $\mathcal{H}$. If the open, bounded set $\mathcal{O}$ is globally recurrent for $\mathcal{H}$, consider the inflated system

$$
\widehat{\mathcal{H}}:=\left(C, F, \mathbb{R}^{n}, \widehat{G}\right),
$$


where $\widehat{G}(x)=G_{1}(x) \cup G_{2}(x)$ with $G_{1}(x)=G(x)$ for $x \in D$ and $G_{1}(x)=\varnothing$ for $x \notin D$, and $G_{2}(x)=x^{*}$ for some $x^{*} \in \mathcal{O}$ and for all $x \in \mathbb{R}^{n}$. From the data of the hybrid system $\widehat{\mathcal{H}}$ and recurrence of the set $\mathcal{O}$ for $\mathcal{H}$, it follows that the maximal solutions of $\widehat{\mathcal{H}}$ are complete.

Lemma 1. The data of the hybrid system $\widehat{\mathcal{H}}$ in (5) satisfies Standing Assumption 1.

Proof. Since $\mathcal{H}$ satisfies Standing Assumption 1, only the outer semicontinuity and local boundedness of $\widehat{G}$ needs to be verified. Since $G_{1}$ and $G_{2}$ are locally bounded, this implies the local boundedness of $\widehat{G}$. The outer semicontinuity of $G_{1}$ follows from outer semicontinuity of $G$ and the set $D$ being closed. The mapping $G_{2}$ is continuous. The outer semicontinuity of $\widehat{G}$ follows from [31, Proposition 2] since it is the union of two outer semicontinuous mappings.

Lemma 2. If the open, bounded set $\mathcal{O}$ is globally recurrent for $\mathcal{H}$ in (2), then $\mathcal{O}$ is globally recurrent for $\widehat{\mathcal{H}}$ in (5).

Proof. Since the flow map for the hybrid system $\widehat{\mathcal{H}}$ is the same as $\mathcal{H}$, the solutions generated by $\widehat{\mathcal{H}}$ do not exhibit finite escape times. Let $\psi$ be any solution to $\widehat{\mathcal{H}}$. If $\psi$ is a solution of $\mathcal{H}$, then there exists $(t, j)$ such that $\psi(t, j) \in \mathcal{O}$. If $\psi$ is not a solution of $\mathcal{H}$, then there exists $(t, j)$ such that $\psi(t, j)=x^{*} \in \mathcal{O}$. Hence global recurrence of $\mathcal{O}$ for $\widehat{\mathcal{H}}$ follows.

The next theorem states that recurrence of an open bounded set $\mathcal{O}$ implies the existence of a smaller recurrent set inside $\mathcal{O}$. This result is primarily used to obtain a smooth Lyapunov function that certifies recurrence of $\mathcal{O}$. A similar result is established for stochastic difference inclusions in [23, Theorem 3].

Theorem 2. If the open, bounded set $\mathcal{O} \subset \mathbb{R}^{n}$ is globally recurrent for $\widehat{\mathcal{H}}$ in (5), then there exists $\varepsilon>0$ and an open set $\widehat{\mathcal{O}}$ satisfying $\widehat{\mathcal{O}}+\varepsilon \mathbb{B}^{o} \subset \mathcal{O}$ such that $\widehat{\mathcal{O}}$ is globally recurrent for $\widehat{\mathcal{H}}$.

Proof. We prove the theorem by contradiction. Suppose there does not exist a smaller globally recurrent set inside $\mathcal{O}$. Then, for every $i \in \mathbb{Z}_{\geq 1}$, there exists a complete solution $\phi_{i}$ such that $\phi_{i}(t, j) \in \mathbb{R}^{n} \backslash \mathcal{O}+1 / i \mathbb{B}$ for all $(t, j) \in \operatorname{dom}\left(\phi_{i}\right)$ and $\phi_{i}\left(\hat{t}_{i}, \hat{j}_{i}\right) \in \mathcal{O}$ for some $\left(\hat{t}_{i}, \hat{j}_{i}\right) \in \operatorname{dom}\left(\phi_{i}\right)$. We now define

$$
t_{i}^{*}:=\inf \left\{t: \phi_{i}(t, j) \in \overline{\mathcal{O}}\right\}, \quad j_{i}^{*}:=\inf \left\{j: \phi_{i}(t, j) \in \overline{\mathcal{O}}\right\}
$$

Define new solutions $\psi_{i}$ such that $\psi_{i}(t, j)=\phi_{i}\left(t+t_{i}^{*}, j+j_{i}^{*}\right)$. Hence $\psi_{i}(0,0) \in \overline{\mathcal{O}}$ for all $i \in \mathbb{Z}_{\geq 1}$ and $\psi_{i}(t, j) \in$ $\left(\mathbb{R}^{n} \backslash \mathcal{O}\right)+(1 / i) \mathbb{B}$ for all $(t, j) \in \operatorname{dom}\left(\psi_{i}\right)$. Since $\psi_{i}(0,0) \in \overline{\mathcal{O}}$, and there are no finite escape times due to recurrence of the set $\mathcal{O}$, it follows from [25, Prop 6.13] that the sequence of solutions $\psi_{i}$ is locally eventually bounded. Then, from [29, Thm 4.4] there exists a subsequence (which we do not relabel) that converges to a solution $\psi$ which is complete since $\psi_{i}$ are complete. The solutions $\psi_{i}$ stay in the closed set $S_{i}:=\left(\mathbb{R}^{n} \backslash \mathcal{O}\right)+1 / i \mathbb{B}$ for all time. Let $(t, j) \in \operatorname{dom}(\psi)$. Then, there exists $\left(t_{i}, j_{i}, \psi_{i}\left(t_{i}, j_{i}\right)\right) \rightarrow(t, j, \psi(t, j))$ with $\psi_{i}\left(t_{i}, j_{i}\right) \in S_{i}$. Then, the limit $\psi(t, j) \in \mathbb{R}^{n} \backslash \mathcal{O}$. Since $(t, j)$ are arbitrary, this implies that the solution $\psi$ stays in the set $\mathbb{R}^{n} \backslash \mathcal{O}$ for all time. This contradicts the global recurrence of the set $\mathcal{O}$. 
The next inflation of the data of $\widehat{\mathcal{H}}$ results in preserving recurrence while slowing down the worst case first hitting time for solutions. This inflation will help in constructing a Lyapunov function that will be radially unbounded. If the open, bounded set $\mathcal{O}$ is globally recurrent for $\widehat{\mathcal{H}}$, let $x^{*} \in \mathcal{O}$ and define the continuous set-valued mapping $M_{\nu}(x):=\left\{x^{*}\right\}+\nu\left(\left|x-x^{*}\right|\right) \mathbb{B}$ where $\nu \in \mathcal{K}_{\infty}$. Consider the inflated mapping

$$
\widehat{\mathcal{H}}_{\nu}:=\left(C, F, \mathbb{R}^{n}, \widehat{G}_{\nu}\right)
$$

where $\widehat{G}(x)=G_{1}(x) \cup M_{\nu}(x)$. The proof of the next lemma is very similar to Lemma 1 .

Lemma 3. For every $\nu \in \mathcal{K}_{\infty}$, the data of the hybrid system $\widehat{\mathcal{H}}_{\nu}$ in (6) satisfies Standing Assumption 1.

The next theorem claims the existence of a $\nu \in \mathcal{K}_{\infty}$ small enough to preserve recurrence of the set $\mathcal{O}$ for the inflated system $\widehat{\mathcal{H}}_{\nu}$ if $\mathcal{O}$ is globally recurrent for $\widehat{\mathcal{H}}$. A similar result is established for stochastic difference inclusions in [23, Theorem 4].

Theorem 3. If the open, bounded set $\mathcal{O} \subset \mathbb{R}^{n}$ is globally recurrent for $\widehat{\mathcal{H}}$ in (5), then there exists $\nu \in \mathcal{K}_{\infty}$ such that $\mathcal{O}$ is globally recurrent for $\widehat{\mathcal{H}}_{\nu}$ in (6).

Proof. Let $S_{i} \subset \mathbb{R}^{n}$ be a sequence of compact sets such that $S_{i} \subset S_{i+1}, \cup_{i \in \mathbb{Z}_{\geq 0}} S_{i}=\mathbb{R}^{n}$ and $S_{0}$ is a small neighborhood of $x^{*}$ that is contained in the set $\mathcal{O}$. It follows from Proposition 2 that global recurrence of $\mathcal{O}$ for $\widehat{\mathcal{H}}$ implies that the reachable set (in infinite hybrid time) from $\mathcal{O}$ is bounded. Uniform global recurrence of $\mathcal{O}$ for $\widehat{\mathcal{H}}$ implies that for every compact set $S_{i}$, there exists a time $J_{i}$ such that solutions from $S_{i}$ for the system $\widehat{\mathcal{H}}$ reach the set $\mathcal{O}$ within time $J_{i}$. Then, the reachable set (in infinite hybrid time) from $S_{i}$ is $\mathcal{R}\left(S_{i}\right):=\mathcal{R}_{\leq J_{i}}\left(S_{i}\right) \cup \Gamma$ where $\mathcal{R}_{\leq J_{i}}\left(S_{i}\right)$ is the reachable set from $S_{i}$ within time $J_{i}$ and $\Gamma$ is the reachable set from $\mathcal{O}$ for the system $\widehat{\mathcal{H}}$. Since both $\Gamma$ and $\mathcal{R}_{\leq J_{i}}\left(S_{i}\right)$ are bounded, $\mathcal{R}\left(S_{i}\right)$ is also bounded. Define $\gamma_{i}:=\sup _{x \in \mathcal{R}\left(S_{i}\right)}\left|x-x^{*}\right|$ and $r_{i}=\inf _{x \in \partial S_{i-1}}\left|x-x^{*}\right|$ for $i \in \mathbb{Z}_{\geq 1}$. Let $\nu \in \mathcal{K}_{\infty}$ be such that for every $i \in \mathbb{Z}_{\geq 1}, \nu\left(\gamma_{i}\right)<r_{i} / 2$.

We now claim that for each $i \in \mathbb{Z}_{\geq 1}$, every solution $\phi \in \mathcal{S}_{\widehat{\mathcal{H}}_{\nu}}\left(S_{i}\right)$ there exists $(t, j) \in \operatorname{dom}(\phi)$ such that $\phi(t, j) \in S_{i-1} \cup \mathcal{O}$. Let a solution $\phi \in \mathcal{S}_{\widehat{\mathcal{H}}_{\nu}}\left(S_{i}\right)$ be given. If the solution $\phi$ can also be generated by $\widehat{\mathcal{H}}$ from the set $S_{i}$, then global recurrence of $\mathcal{O}$ for $\widehat{\mathcal{H}}$ implies that there exists $(t, j) \in \operatorname{dom}(\phi)$ such that $\phi(t, j) \in \mathcal{O}$. If $\phi$ is not a solution generated by $\widehat{\mathcal{H}}$, then there exists $(t, j) \in \operatorname{dom}(\phi)$ such that $(t, j)$ is the first jump time satisfying $\phi(t, j+1) \in\left\{x^{*}\right\}+\nu\left(|\phi(t, j)|-x^{*}\right)$. Then, necessarily $\phi(t, j) \in \mathcal{R}\left(S_{i}\right)$. From the construction of the mapping $\nu$, it follows that $\phi(t, j+1) \in S_{i-1}$. This establishes the claim.

We now establish that for every solution $\phi \in \mathcal{S}_{\widehat{\mathcal{H}}_{\nu}}\left(\mathbb{R}^{n}\right)$, there exists $(t, j) \in \operatorname{dom}(\phi)$ such that $\phi(t, j) \in \mathcal{O}$. Let the $\phi \in \mathcal{S}_{\widehat{\mathcal{H}}_{\nu}}\left(\mathbb{R}^{n}\right)$ be given. Then, there exists $i \in \mathbb{Z}_{\geq 1}$ such that $\phi(0,0) \in S_{i}$. If $\phi$ is a solution of $\widehat{\mathcal{H}}$, the result follows from global recurrence of $\mathcal{O}$ for $\mathcal{H}$. If $\phi$ is not a solution of $\widehat{\mathcal{H}}$, it follows from the above claim that there exists $\left(t_{i}, j_{i}\right) \in \operatorname{dom}(\phi)$ such that $\phi\left(t_{i}, j_{i}\right) \in S_{i-1}$. We now apply the result of the above claim in an iterative manner from the set $S_{i-1}$ till the solution $\phi$ reaches the set $S_{0}$. Hence, there exists positive constants $\left\{t_{k}\right\}_{k=0}^{i-1}$ and $\left\{j_{k}\right\}_{k=0}^{i-1}$ such that $\phi\left(\sum_{k=j}^{i} t_{k}, \sum_{k=j}^{i} j_{k}\right) \in S_{j-1}$ for every $j \in\{1, \ldots, i\}$. Since $S_{0} \subset \mathcal{O}$, it follows that $\phi\left(\sum_{k=1}^{i} t_{k}, \sum_{k=1}^{i} j_{k}\right) \in S_{0} \subset \mathcal{O}$. This establishes global recurrence of the set $\mathcal{O}$ for the hybrid system $\widehat{\mathcal{H}}_{\nu}$. 
Finally, we introduce state dependent perturbations typically used in robustness analysis. For a continuous, positive valued function $\delta: \mathbb{R}^{n} \rightarrow \mathbb{R}_{>0}$, we denote the perturbed version of $\widehat{\mathcal{H}}$ by

$$
\widehat{\mathcal{H}}_{\delta}:=\left(C_{\delta}, F_{\delta}, \mathbb{R}^{n}, \widehat{G}_{\delta}\right),
$$

where

$$
\begin{aligned}
C_{\delta} & :=\left\{x \in \mathbb{R}^{n}:(x+\delta(x) \mathbb{B}) \cap C \neq \varnothing\right\} \\
F_{\delta} & :=\overline{\operatorname{con}} F((x+\delta(x) \mathbb{B}) \cap C)+\delta(x) \mathbb{B} \\
\widehat{G}_{\delta} & :=\left\{v \in \mathbb{R}^{n}: v \in g+\delta(g) \mathbb{B}, g \in \widehat{G}(x+\delta(x) \mathbb{B})\right\}
\end{aligned}
$$

and $\overline{c o n}$ refers to the closed convex hull. The next result follows from [25, Proposition 6.28].

Lemma 4. For every continuous $\delta: \mathbb{R}^{n} \rightarrow \mathbb{R}_{>0}$, the data of the hybrid system $\widehat{\mathcal{H}}_{\delta}$ in (7) satisfies Standing Assumption 1.

We establish that the recurrence property is robust is to sufficiently small state dependent perturbation. So under the regularity conditions in Standing Assumption 1 if there exists an open, bounded set that is globally recurrent for the nominal system, then there exists a sufficiently small perturbation such that the recurrence property is preserved for the inflated system $\widehat{\mathcal{H}}_{\delta}$ in (7). The following lemma will be used to construct the state dependent perturbation in the robustness result.

Lemma 5. Let the open, bounded set $\mathcal{O}$ be globally recurrent for $\widehat{\mathcal{H}}$ in (5). Then, for each compact set $K$, there exists $\delta>0$ such that every solution from the set $K$ reaches the set $\mathcal{O}$ for $\widehat{\mathcal{H}}_{\delta}$ in (7).

Proof. We establish the result by contradiction. Suppose the statement of the lemma is not true, then there exists a compact set $K$ such that for every $i \in \mathbb{Z}_{\geq 1}$, there exists a solution $\phi_{i} \in \mathcal{S}_{\widehat{\mathcal{H}}_{1 / i}}(K)$ such that $\phi_{i}(t, j) \notin \mathcal{O}$ for every $(t, j) \in \operatorname{dom}\left(\phi_{i}\right)$. Since the hybrid system is well posed [25, Def 6.29, Thm 6.30], it follows from [25, Thm 6.1, Prop 6.33] that the sequence of solutions $\phi_{i}$ has a subsequence which we do not relabel that converges to the solution $\phi \in \mathcal{S}_{\widehat{\mathcal{H}}}(K)$ for the nominal system. Since the set $\mathcal{O}$ is globally recurrent for the nominal system, there exists $(t, j)$ such that $\phi(t, j) \in \mathcal{O}$. Then from convergence of hybrid arcs, there exists a sequence $\left(t_{i}, j_{i}, \phi_{i}\left(t_{i}, j_{i}\right)\right)$ that converges to $(t, j, \phi(t, j))$. Then, since the set $\mathcal{O}$ is open, for large enough $i, \phi_{i}\left(t_{i}, j_{i}\right) \in \mathcal{O}$, which contradicts the initial assumption.

Theorem 4. If the open, bounded set $\mathcal{O} \subset \mathbb{R}^{n}$ is globally recurrent for $\widehat{\mathcal{H}}$ in (5), then there exists a continuous function $\delta: \mathbb{R}^{n} \rightarrow \mathbb{R}_{>0}$ such that $\mathcal{O}$ is globally recurrent for $\widehat{\mathcal{H}}_{\delta}$ in (7).

Proof. It follows from Proposition 4 that global recurrence of $\mathcal{O}$ for $\widehat{\mathcal{H}}$ implies that there exists a compact set $\mathcal{A}$ that is uniformly globally asymptotically stable for $\widehat{\mathcal{H}}$. Then, from [25, Thm 7.21], it follows that there exists a continuous function $\rho_{1}: \mathbb{R}^{n} \rightarrow \mathbb{R}_{\geq 0}$ that is zero on the set $\mathcal{A}$ and positive elsewhere such that $\mathcal{A}$ is uniformly globally asymptotically stable for $\widehat{\mathcal{H}}_{\rho_{1}}$. Let $\varepsilon \in(0,1)$. It follows from [25, Lemma 7.20] that there exists a constant 
$\rho_{2}>0$ such that solutions of $\widehat{\mathcal{H}}_{\rho_{2}}$ from the compact set $\mathcal{A}+\mathbb{B}$ converge to $\mathcal{A}+\varepsilon \mathbb{B}$. Now choose a continuous function $\rho: \mathbb{R}^{n} \rightarrow \mathbb{R}_{>0}$ such that $\rho(x) \leq \rho_{2}$ for $x \in \mathcal{A}+\mathbb{B}$ and $\rho(x) \leq \min \left\{\rho_{1}(x), \rho_{2}\right\}$ otherwise. Then, it follows that solutions of the system $\widehat{\mathcal{H}}_{\rho}$ reach the open set $\mathcal{A}+\mathbb{B}^{o}$.

Let $\delta_{1}>0$ satisfy the condition of Lemma 5 for the compact set $K=\mathcal{A}+\mathbb{B}$. Let $\delta: \mathbb{R}^{n} \rightarrow \mathbb{R}_{>0}$ be a continuous function which satisfies $\delta(x) \leq \min \left\{\delta_{1}, \rho(x)\right\}$. Let $\phi \in \mathcal{S}_{\widehat{\mathcal{H}}_{\delta}}\left(\mathbb{R}^{n}\right)$. Since $\delta(x) \leq \rho(x)$ for all $x \in \mathbb{R}^{n}$, it follows that there exists $\left(t_{1}, j_{1}\right) \in \operatorname{dom}(\phi)$ such that $\phi\left(t_{1}, j_{1}\right) \in \mathcal{A}+\mathbb{B}^{o}$. Define a solution $\psi(t, j):=\phi\left(t_{1}+t, j_{1}+j\right)$ for all $(t, j)$ such that $\left(t_{1}+t, j_{1}+j\right) \in \operatorname{dom}(\phi)$. From Lemma 5 there exists $\left(t_{2}, j_{2}\right) \in \operatorname{dom}(\psi)$ such that $\psi\left(t_{2}, j_{2}\right) \in \mathcal{O}$. Hence, we have $\phi\left(t_{1}+t_{2}, j_{1}+j_{2}\right) \in \mathcal{O}$. This establishes global recurrence of the set $\mathcal{O}$ for $\widehat{\mathcal{H}}_{\delta}$.

The following example illustrates that recurrence is not necessarily robust to arbitrarily small perturbations if the conditions of Standing Assumption 1 are not satisfied.

Example 4: Consider the system in Example 1. The Krasovskii regularization of the mapping $g$ (the smallest closed set that contains all the limit points of $g)$ defined as $G(x):=\cap_{\rho>0} \overline{g(x+\rho \mathbb{B})}$ is set-valued at the point of discontinuity $x=1$. So for $x=1$ we have $G(1)=\{0,1\}$. Hence, when the set $\mathcal{O}$ is a small neighborhood of the origin, the point $x=1$ becomes a fixed point and hence even for arbitrarily small perturbations, the recurrence property fails and consequently the set $\mathcal{O}$ is not robustly recurrent for $\mathcal{H}$.

The next example shows that the recurrence property is not necessarily robust if the set $\mathcal{O}$ is not open.

Example 5: Consider the simple discrete-time system $x^{+}=0$ for $x \in \mathbb{R}$. Let the set $\mathcal{O}=\{0\}$. Global recurrence of the set $\mathcal{O}$ follows from the system dynamics. Let $\delta: \mathbb{R} \rightarrow \mathbb{R}_{>0}$ be any continuous function. Then, the perturbed system is represented as $x^{+} \in \delta(0) \mathbb{B}$. Since $\delta(0)>0$, it is clear that not all solutions of the perturbed system reach the set $\mathcal{O}$ and hence the set $\mathcal{O}$ is not robustly recurrent.

\section{B. Converse Lyapunov theorem for recurrence}

We now present a Lyapunov function based characterization of recurrence for open, bounded sets. A smooth function $V: \mathbb{R}^{n} \rightarrow \mathbb{R}_{\geq 0}$ is a Lyapunov function with respect to the set $\mathcal{O}$ for $\mathcal{H}$ if it is radially unbounded and there exists $\mu>0$ such that

$$
\begin{aligned}
\langle\nabla V(x), f\rangle & \leq-1+\mu \mathbb{I}_{\mathcal{O}}(x), \forall x \in C, f \in F(x) \\
V(g)-V(x) & \leq-1+\mu \mathbb{I}_{\mathcal{O}}(x), \forall x \in D, g \in G(x) .
\end{aligned}
$$

In essence the Lyapunov function in (8) and (9) satisfies a strict decrease condition along solutions outside the set $\mathcal{O}$ as opposed to the non-strict decrease conditions in (3) and (4). The robustness results established in the previous section are utilized to establish a converse Lyapunov theorem for recurrence of an open, bounded set for the hybrid system $\mathcal{H}$.

The outline of the construction of the Lyapunov function used in the converse theorem is as follows. Under the assumption that an open, bounded set $\mathcal{O}$ is globally recurrent for $\mathcal{H}$ in (2), we apply the robustness results in Section VI.A to establish that $\widehat{\mathcal{O}} \subset \mathcal{O}+\varepsilon \mathbb{B}^{o}$ is globally recurrent for the system $\widehat{\mathcal{H}}_{\nu, \delta}$ for some $\nu \in \mathcal{K}_{\infty}, \varepsilon>0$ 
and a continuous, positive function $\delta$. The system $\widehat{\mathcal{H}}_{\nu, \delta}$ is a perturbed version of the system $\widehat{\mathcal{H}}_{\nu}$ in $(6)$. We then construct a preliminary (possibly non-smooth) Lyapunov function $V_{0}$ to certify recurrence of the set $\widehat{\mathcal{O}}$ for $\widehat{\mathcal{H}}_{\nu, \delta}$. The construction of $V_{0}$ is related to worst-case first hitting for solutions to the set $\mathcal{O}$. A similar construction is used for discrete-time systems in [15]. The function $V_{0}$ is then smoothed to arrive at a smooth Lyapunov function $V$ to certify recurrence of $\mathcal{O}$ for the system $\mathcal{H}$ in (2). A similar outline for establishing a converse Lyapunov theorem is used in [13] and [10].

We now state a necessary and sufficient condition for global recurrence of an open, bounded set for $\mathcal{H}$. The proof of the following theorem is in Section VIII.

Theorem 5. The open, bounded set $\mathcal{O} \subset \mathbb{R}^{n}$ is globally recurrent for $\mathcal{H}$ in (2) if and only if there exists a smooth Lyapunov function relative to $\mathcal{O}$ for $\mathcal{H}$.

The following result is a simple corollary of Theorem 5 and Proposition 2 that establishes the equivalence between the solutions of $\mathcal{H}$ being uniformly ultimately bounded and the existence of a smooth Lyapunov function that satisfies in (8) and (9) with respect to an open, bounded set $\mathcal{O}$.

Corollary 1. The solutions of $\mathcal{H}$ in (2) are uniformly ultimately bounded if and only if there exists an open, bounded set $\mathcal{O}$ and a smooth Lyapunov function that satisfies (8) and (9) with respect to $\mathcal{O}$ for $\mathcal{H}$.

The following examples illustrates that the existence of even a continuous Lyapunov functions is not necessarily guaranteed without the conditions of Standing Assumption 1.

Example 6: Consider the system in Example 1. We show that there does not exist a continuous Lyapunov function with respect to the set $\mathcal{O}=(-\varepsilon, \varepsilon)$ for any $\varepsilon \in(0,1)$. We establish the claim by contradiction. Suppose $V$ is a continuous Lyapunov function. Then, $V(g(x)) \leq V(x)-1$ for $x \in \mathbb{R}^{n} \backslash \mathcal{O}$. Let $x_{i} \in[\varepsilon, 1)$ be such that $\lim _{i \rightarrow \infty} x_{i}=1$. Then $\lim _{i \rightarrow \infty} g\left(x_{i}\right)=1$ even though $g(1)=0$. Then, $\lim _{i \rightarrow \infty} V\left(g\left(x_{i}\right)\right)-V\left(x_{i}\right)=0$, which contradicts the strict decrease condition of $V$. Hence, there does not exist a continuous Lyapunov function for the system. This is essentially due to the non-robust nature of recurrence as explained in Example 4.

\section{HitTING TIME TO OPEN SETS - AN EQUIVALENT CHARACTERIZATION}

We now establish an equivalent characterization for recurrence in terms of functions that will be used to construct the Lyapunov function in the converse theorem. Let $\mathcal{O} \subset \mathbb{R}^{n}$ be an open set. Let $\widetilde{\mathcal{H}}$ be a hybrid system whose data satisfies the conditions of Standing Assumption 1, and for which the maximal solutions are complete. We define for every hybrid arc $\phi \in \mathcal{S}_{\widetilde{\mathcal{H}}}\left(\mathbb{R}^{n}\right)$ the function

$$
W^{\mathcal{O}}(\phi):=\inf _{(t, j) \in \operatorname{dom}(\phi), \phi(t, j) \in \mathcal{O}}(t+j) .
$$

For every $\phi, W^{\mathcal{O}}(\phi)$ is related to the first time the solution $\phi$ hits the set $\mathcal{O}$. If the solution $\phi$ never hits the set $\mathcal{O}$, then $W^{\mathcal{O}}(\phi)=\infty$. The worst first hitting time to the set $\mathcal{O}$ from the initial condition $x$ for the system $\tilde{\mathcal{H}}$ is then related to the quantity $\sup _{\phi \in \mathcal{S}_{\widetilde{\mathcal{H}}}(x)} W^{\mathcal{O}}(\phi)$. 
The following result then establishes the connection between global recurrence and the first hitting times and the proof follows from the definition of recurrence and Proposition 1.

Proposition 5. Let $\mathcal{O} \subset \mathbb{R}^{n}$ be open and bounded. Then, the following statements are equivalent.

1) $\mathcal{O}$ is globally recurrent for $\widetilde{\mathcal{H}}$.

2) $\mathcal{O}$ is uniformly globally recurrent $\widetilde{\mathcal{H}}$.

3) For every compact set $K \subset \mathbb{R}^{n}$, there exists $T_{K}>0$ such that $\sup _{\phi \in \mathcal{S}_{\widetilde{\mathcal{H}}}(K)} W^{\mathcal{O}}(\phi) \leq T_{K}$.

4) For every $x \in \mathbb{R}^{n}$ and $\phi \in \mathcal{S}_{\widetilde{\mathcal{H}}}(x), W^{\mathcal{O}}(\phi)<\infty$.

Proof. 1) $\Rightarrow 2$ ) The implication follows from Proposition 1.2) $\Rightarrow 3$ ) Since the set $\mathcal{O}$ is uniformly globally recurrent, this implies that for every compact $K$, there exists $T_{K}>0$ such that for every $\phi \in \mathcal{S}_{\widetilde{\mathcal{H}}}(K)$ there exists $(t, j)$ such that $\phi(t, j) \in \mathcal{O}$ and $t+j \leq T_{K}$. This implies that for every $\left.\left.\phi \in \mathcal{S}_{\widetilde{\mathcal{H}}}(K), W^{\mathcal{O}}(\phi) \leq T_{K} .3\right) \Rightarrow 4\right)$ This implication is trivial. 4) $\Rightarrow 1$ ) For any $\phi$ such that $W^{\mathcal{O}}(\phi)<\infty$ and every $\varepsilon>0$ there exists $(t, j)$ such that $t+j \leq W^{\mathcal{O}}(\phi)+\varepsilon$ and $\phi(t, j) \in \mathcal{O}$. This establishes global recurrence of the set $\mathcal{O}$.

From now on we will use (10) only to characterize recurrence in hybrid systems for which the maximal solutions are complete. Hence, in the later sections the results of Proposition 5 will be applied to $\widehat{\mathcal{H}}, \widehat{\mathcal{H}}_{\nu}$ or $\widehat{\mathcal{H}}_{\delta}$. The next result establishes that the worst case hitting time to open sets is an upper semicontinuous function.

Proposition 6. If the open, bounded set $\mathcal{O} \subset \mathbb{R}^{n}$ is globally recurrent for $\widetilde{\mathcal{H}}$, then the mapping $x \mapsto$ $\sup _{\phi \in \mathcal{S}_{\widetilde{\mathcal{H}}}(x)} W^{\mathcal{O}}(\phi)$ is well defined and upper semicontinuous.

Proof. Local boundedness of the mapping follows from Propositions 1 and 5 and hence, the mapping $x \mapsto$ $\sup _{\phi \in \mathcal{S}_{\widetilde{\mathcal{H}}}(x)} W^{\mathcal{O}}(\phi)$ is well defined. Let $\phi_{i}$ be a sequence of hybrid arcs that converges graphically to the hybrid $\operatorname{arc} \phi$. We will establish that $\limsup _{i \rightarrow \infty} W^{\mathcal{O}}\left(\phi_{i}\right) \leq W^{\mathcal{O}}(\phi)$. Let $\varepsilon>0$ be arbitrary. Let $(t, j)$ be such that $t+j \leq W^{\mathcal{O}}(\phi)+\varepsilon / 2$ and $\phi(t, j) \in \mathcal{O}$. Since $\phi_{i}$ converge to $\phi$ graphically, there exist sequences $\left(t_{i}, j_{i}\right) \rightarrow(t, j)$ and $\phi_{i}\left(t_{i}, j_{i}\right) \rightarrow \phi(t, j)$. Since $\mathcal{O}$ is open, for $i$ large enough $\phi_{i}\left(t_{i}, j_{i}\right) \in \mathcal{O}$. Hence, without loss of generality for $i$ large enough we have $W^{\mathcal{O}}\left(\phi_{i}\right) \leq t_{i}+j_{i}$ and $t_{i}+j_{i} \leq t+j+\varepsilon / 2$. This implies that

$$
\limsup _{i \rightarrow \infty} W^{\mathcal{O}}\left(\phi_{i}\right) \leq t_{i}+j_{i} \leq t+j+\varepsilon / 2 \leq W^{\mathcal{O}}(\phi)+\varepsilon
$$

Since $\varepsilon>0$ is arbitrary, it follows that $\lim _{\sup _{i \rightarrow \infty}} W^{\mathcal{O}}\left(\phi_{i}\right) \leq W^{\mathcal{O}}(\phi)$. The upper semicontinuity of the mapping $x \mapsto \sup _{\phi \in \mathcal{S}_{\widetilde{\mathcal{H}}}(x)} W^{\mathcal{O}}(\phi)$ follows directly from the proof of [28, Lemma 8.3] using [29, Thm 4.4].

\section{Proof OF THEOREM 5}

\section{A. Necessity}

1) Preliminary Lyapunov function: In this section, we will construct a Lyapunov function for an inflated system which while not necessarily smooth satisfies good regularity properties and decrease conditions. The robustness results from the previous sections will then be used to establish the smoothness of the Lyapunov function. 
Let the open, bounded set $\mathcal{O} \subset \mathbb{R}^{n}$ be globally recurrent for the system $\mathcal{H}$. Then from Lemma $1, \mathcal{O}$ is globally recurrent for the system $\widehat{\mathcal{H}}$. From Theorem 2, there exists a smaller open set $\widehat{\mathcal{O}}_{2}$ such that $\widehat{\mathcal{O}}_{2}+\varepsilon_{2} \mathbb{B}^{o} \subset \mathcal{O}$ for some $\varepsilon_{2}>0$.

From Theorem 3, there exists a $\mathcal{K}_{\infty}$ function $\nu$ such that $\widehat{\mathcal{O}}_{2}$ is globally recurrent for $\widehat{\mathcal{H}}_{\nu}$. Finally, from Theorem 4 , there exists a continuous state dependent perturbation $\delta: \mathbb{R}^{n} \rightarrow \mathbb{R}_{>0}$ such that $\widehat{\mathcal{O}}_{2}$ is globally recurrent for $\widehat{\mathcal{H}}_{\nu, \delta}$. We will construct a preliminary Lyapunov function using the solutions of the system $\widehat{\mathcal{H}}_{\nu, \delta}$. Define $V_{0}: \mathbb{R}^{n} \rightarrow \mathbb{R}_{\geq 0}$ as

$$
V_{0}(x):=\sup _{\phi \in \mathcal{S}_{\widehat{\mathcal{H}}_{\nu, \delta}}(x)} W^{\widehat{\mathcal{O}}_{2}}(\phi)
$$

Proposition 7. The function $V_{0}$ is radially unbounded and upper semicontinuous.

Proof. The upper semicontinuity of $V_{0}$ follows from Proposition 6. We just need to establish the radial unboundedness of the function $V_{0}$. Let $Q_{i}=\overline{\widehat{\mathcal{O}}_{2}+(i+1) \mathbb{B}^{o}} \backslash\left(\widehat{\mathcal{O}}_{2}+i \mathbb{B}^{o}\right)$ be a sequence of compact sets for $i \in \mathbb{Z}_{\geq 0}$. We now consider the solutions of the system $x^{+} \in M_{\nu}(x)=x^{*}+\nu\left(\left|x-x^{*}\right|\right) \mathbb{B}$ for $x \in \mathbb{R}^{n}$. It can be observed that solutions generated by $M_{\nu}$ are a subset of the solutions generated by $\widehat{\mathcal{H}}_{\nu, \delta}$. Let $\alpha_{i} \in \mathbb{R}_{>0}$ for each $i \in \mathbb{Z}_{\geq 0}$ be such that $\alpha_{i}=\inf _{x \in Q_{i}} \sup _{\phi \in \mathcal{S}_{M_{\nu}}(x)} W^{\widehat{\mathcal{O}}_{2}}(\phi)$. Since $\nu \in \mathcal{K}_{\infty}$, it follows from the structure of $Q_{i}$ and $M_{\nu}$ that the mapping $i \mapsto \alpha_{i}$ is increasing and unbounded. Then the worst first hitting times generated by solutions hybrid system $\widehat{\mathcal{H}}_{\nu, \delta}$ necessarily satisfies $\alpha_{i} \leq \sup _{\phi \in \mathcal{S}_{\widehat{\mathcal{H}}_{\nu, \delta}}(x)} W^{\widehat{\mathcal{O}}_{2}}(\phi)$ for $x \in Q_{i}$. As $i \rightarrow \infty$, then it follows that $V_{0}$ is radially unbounded.

Proposition 8. Let $\phi \in \mathcal{S}_{\widehat{\mathcal{H}}_{\nu, \delta}}(x)$ be such that $\operatorname{graph}(\phi) \cap\left([0, t] \times\{0, \ldots, \max \{0, j-1\}\} \times \mathbb{R}^{n}\right) \subset \mathbb{R}^{2} \times \mathbb{R}^{n} \backslash \widehat{\mathcal{O}}_{2}$ for some $(t, j) \in \operatorname{dom}(\phi)$ then

$$
V_{0}(\phi(t, j)) \leq V_{0}(x)-(t+j)
$$

Proof. Let $\hat{\phi}^{*} \in \mathcal{S}_{\widehat{\mathcal{H}}_{\nu, \delta}}(\phi(t, j))$ be a solution such that $W^{\widehat{\mathcal{O}}_{2}}\left(\hat{\phi}^{*}\right)=\sup _{\hat{\phi} \in \mathcal{S}_{\widehat{\mathcal{H}}_{\nu, \delta}}(\phi(t, j))} W^{\widehat{\mathcal{O}}_{2}}(\hat{\phi})$. Such a solution $\hat{\phi}^{*}$ exists due to regularity properties of hitting time function $W^{\widehat{\mathcal{O}_{2}}}$ and similar reasoning used in the proof of [28, Lemma 8.3]. Now let $\phi^{*} \in \mathcal{S}_{\widehat{\mathcal{H}}_{\nu, \delta}}(x)$ be a solution satisfying $\phi^{*}(\tilde{t}, \tilde{j})=\phi(\tilde{t}, \tilde{j})$ for $\tilde{t} \leq t, \tilde{j} \leq j$ and $\phi^{*}(t+\tilde{t}, j+\tilde{j})=\hat{\phi}^{*}(\tilde{t}, \tilde{j})$ otherwise. We consider two cases depending on $\phi(t, j)$. First, let $\phi(t, j) \in \mathbb{R}^{n} \backslash \widehat{\mathcal{O}}_{2}$. Then, it can observed that $W^{\widehat{\mathcal{O}}_{2}}\left(\phi^{*}\right)=W^{\widehat{\mathcal{O}}_{2}}\left(\hat{\phi}^{*}\right)+t+j$. Next, we note that if $\phi(t, j) \in \widehat{\mathcal{O}}_{2}$, then from the assumptions on the solution $\phi$ we have $W^{\widehat{\mathcal{O}}_{2}}\left(\hat{\phi^{*}}\right)=0, W^{\widehat{\mathcal{O}}_{2}}\left(\phi^{*}\right)=t+j$ and $W^{\widehat{\mathcal{O}}_{2}}\left(\phi^{*}\right)=W^{\widehat{\mathcal{O}}_{2}}\left(\hat{\phi}^{*}\right)+t+j$. Hence, it follows that

$$
\begin{aligned}
V_{0}(\phi(t, j)) & =\sup _{\hat{\phi} \in \mathcal{S}_{\widehat{\mathcal{H}}_{\nu, \delta}}(\phi(t, j))} W^{\widehat{\mathcal{O}}_{2}}(\hat{\phi}) \\
& =W^{\widehat{\mathcal{O}}_{2}}\left(\hat{\phi}^{*}\right)=W^{\widehat{\mathcal{O}}_{2}}\left(\phi^{*}\right)-t-j \\
& \leq \sup _{\phi \in \mathcal{S}_{\widehat{\mathcal{H}}_{\nu, \delta}}(x)} W^{\widehat{\mathcal{O}}_{2}}(\phi)-(t+j)=V_{0}(x)-(t+j) .
\end{aligned}
$$


2) Smoothing of Lyapunov function: Now choose the open set $\widehat{\mathcal{O}}_{1}$ such that $\widehat{\mathcal{O}}_{2}+\left(\varepsilon_{2} / 3\right) \mathbb{B}^{o} \subset \widehat{\mathcal{O}}_{1}$ and $\widehat{\mathcal{O}}_{1}+$ $\left(\varepsilon_{2} / 3\right) \mathbb{B}^{o} \subset \mathcal{O}$. It follows that $\widehat{\mathcal{O}}_{1}$ is also globally recurrent for $\widehat{\mathcal{H}}_{\nu, \delta}$. Define $\rho(x):=\min \left\{\delta(x), \varepsilon_{2} / 3\right\}$. Let $\varrho$ come from [25, Lemma 7.37] using $\rho$. Then, the function $\varrho$ is continuous and positive on bounded sets. We can also conclude that if $x \in \mathbb{R}^{n} \backslash \widehat{\mathcal{O}}_{1}$, then $x+\varrho(x) \mathbb{B} \subset \mathbb{R}^{n} \backslash \widehat{\mathcal{O}}_{2}$. Let $\Psi: \mathbb{R}^{n} \rightarrow[0,1]$ be any infinitely differentiable function such that $\Psi(x)=0$ for $x \notin \mathbb{B}$ and $\int \Psi(x) d x=1$.

Now define $V(x):=\int_{\mathbb{R}^{n}} V_{0}(x+\varrho(x) \eta) \Psi(\eta) d \eta$. The local boundedness and radial unboundedness follows from the properties of $V_{0}$. The smoothness of $V$ on $\mathbb{R}^{n}$, follows from the results in [25, Section 7.36].

From [25, Lemma 7.37], for every $\phi \in \mathcal{S}_{\widehat{\mathcal{H}}_{\nu, e}}\left(\mathbb{R}^{n}\right)$ and $\eta \in \mathbb{B}$, there exists $\psi_{\eta} \in \mathcal{S}_{\widehat{\mathcal{H}}_{\nu, \rho}}\left(\mathbb{R}^{n}\right)$ such that $\operatorname{dom}(\phi)=$ $\operatorname{dom}(\psi), \psi_{\eta}(0,0)=\phi(0,0)+\varrho(\phi(0,0)) \eta$ and $\psi_{\eta}(t, j)=\phi(t, j)+\varrho(\phi(t, j)) \eta$. Let $\phi$ be solution of $\widehat{\mathcal{H}}_{\nu, \varrho}$ from $x$ such that $\operatorname{graph}(\phi) \cap\left([0, t] \times\{0, \ldots, \max \{0, j-1\}\} \times \mathbb{R}^{n}\right) \subset \mathbb{R}^{2} \times \mathbb{R}^{n} \backslash \widehat{\mathcal{O}}_{1}$ for some $(t, j) \in \operatorname{dom}(\phi)$. It follows from the properties of $\varrho$, [25, Lemma 7.37] and Proposition 8 that

$$
\begin{aligned}
V(\phi(t, j)) & =\int_{\mathbb{R}^{n}} V_{0}(\phi(t, j)+\varrho(\phi(t, j)) \eta) \Psi(\eta) d \eta=\int_{\mathbb{R}^{n}} V_{0}\left(\psi_{\eta}(t, j)\right) \Psi(\eta) d \eta \\
& \leq \int_{\mathbb{R}^{n}} V_{0}\left(\psi_{\eta}(0,0)\right) \Psi(\eta) d \eta-(t+j) \\
& =\int_{\mathbb{R}^{n}} V_{0}(x+\varrho(x) \eta) \Psi(\eta) d \eta-(t+j)=V(x)-(t+j) .
\end{aligned}
$$

Then, from [12, Claim 6.3] and (13) it follows that for every $x \in C \cap\left(\mathbb{R}^{n} \backslash \mathcal{O}\right)$ and $f \in F(x)$ and small $t \geq 0, V(x+t f) \leq V(x)-t$. The smoothness of $V$ implies that for every $x \in C \cap\left(\mathbb{R}^{n} \backslash \mathcal{O}\right)$ and $f \in F(x)$ $\langle\nabla V(x), f\rangle \leq-1$. Similarly for $x \in D \cap\left(\mathbb{R}^{n} \backslash \mathcal{O}\right), \phi(0,1)=g$ we have $V(g) \leq V(x)-1$ for $g \in G(x)$.

Finally, we establish the existence of $\mu>0$ such that (8) and (9) hold. Since $V$ is smooth, $\mathcal{O}$ is bounded, and $F$ is locally bounded, there exists $\mu_{1}>0$ such that $\langle\nabla V(x), f\rangle \leq \mu_{1}$ for all $x \in C \cap \mathcal{O}$ and $f \in F(x)$. Similarly local boundedness of $G$ implies the existence of $\mu_{2}>0$ such that $\max _{g \in G(x)} V(g)-V(x) \leq \mu_{2}$ for all $x \in D \cap \mathcal{O}$. Then conditions (8) and (9) hold with $\mu=\max \left\{\mu_{1}, \mu_{2}\right\}$.

\section{B. Sufficiency}

The proof of sufficiency follows from observing that the existence of a $V$ satisfying (8) and (9) satisfies the conditions of Theorem 1.

\section{COnClusions}

A converse Lyapunov theorem for a weak property called recurrence is established for a class of hybrid systems. Robustness of recurrence to sufficiently small state dependent perturbations is established. It is also established that recurrence of open, bounded sets for hybrid systems is equivalent to the well studied property of uniform ultimate boundedness. Future work will involve extending the results in this paper to develop converse Lyapunov theorems for a class of stochastic hybrid systems studied in [28]. 


\section{Appendix: Proof of Proposition 4}

Since $\mathcal{O}$ is recurrent for $\mathcal{H}$, it follows from Lemma 2 that $\mathcal{O}$ is recurrent for the modified system $\widehat{\mathcal{H}}$. From Proposition 2 it follows that solutions of $\widehat{\mathcal{H}}$ are ultimately bounded with ultimate bound $M$. Let $S=(M+1) \mathbb{B}$. Then, there exists $T>0$ such that for $\phi \in \mathcal{S}_{\widehat{\mathcal{H}}}(S)$ if $(t, j) \in \operatorname{dom} \phi$ satisfies $t+j \geq T$, then $\phi(t, j) \in M \mathbb{B}$. Then, it follows that $\Omega(S) \subset M \mathbb{B} \subset \operatorname{int}(S)$. Since the maximal solutions of $\widehat{\mathcal{H}}$ are complete $\Omega(S)$ is non-empty. Then, from [25, Corollary 7.7], it follows that $\Omega(S)$ is compact and asymptotically stable with basin of attraction $S$. Since every solution eventually enters the set $S$, it follows that $\Omega(S)$ is uniformly globally asymptotically stable for $\widehat{\mathcal{H}}$. Since the solutions of $\mathcal{H}$ are also solutions of $\widehat{\mathcal{H}}$, it follows that $\mathcal{A}:=\Omega(S)$ is UGAS for $\mathcal{H}$.

\section{REFERENCES}

[1] R. Goebel, R. G. Sanfelice, and A. R. Teel, "Hybrid dynamical systems," IEEE Control Systems Magazine, vol. 29, no. 2, pp. 28-93, 2009.

[2] P. Collins, "A trajectory-space approach to hybrid systems," in Proc. 16th MTNS, 2004.

[3] M. S. Branicky, V. S. Borkar, and S. K. Mitter, "A unified framework for hybrid control: Model and optimal control theory," IEEE Transactions on Automatic Control, vol. 43, no. 1, pp. 31-45, 1998.

[4] M. Vidyasagar, Nonlinear systems analysis. SIAM, 2002, vol. 42.

[5] H. K. Khalil, Nonlinear systems, 3rd ed. Prentice hall Upper Saddle River, 2002.

[6] Y. Lin, E. D. Sontag, and Y. Wang, "A smooth converse Lyapunov theorem for robust stability," SIAM Journal on Control and Optimization, vol. 34, no. 1, pp. 124-160, 1996.

[7] F. H. Clarke, Y. S. Ledyaev, and R. J. Stern, "Asymptotic stability and smooth Lyapunov functions," Journal of Differential Equations, vol. 149, no. 1, pp. 69-114, 1998.

[8] A. R. Teel and L. Praly, "A smooth Lyapunov function from a class-KL estimate involving two positive semidefinite functions," ESAIM: Control, Optimisation and Calculus of Variations, vol. 5, pp. 313-367, 2000.

[9] Z.-P. Jiang and Y. Wang, "A converse Lyapunov theorem for discrete-time systems with disturbances," Systems \& Control Letters, vol. 45, no. 1 , pp. 49-58, 2002.

[10] C. M. Kellett and A. R. Teel, "Smooth Lyapunov functions and robustness of stability for difference inclusions," Systems \& Control Letters, vol. 52, no. 5, pp. 395-405, 2004.

[11] _ _ "On the robustness of KL-stability for difference inclusions: Smooth discrete-time Lyapunov functions," SIAM Journal on Control and Optimization, vol. 44, no. 3, pp. 777-800, 2005.

[12] C. Cai, A. R. Teel, and R. Goebel, "Smooth Lyapunov functions for hybrid systems Part I: existence is equivalent to robustness," IEEE Transactions on Automatic Control, vol. 52, no. 7, pp. 1264-1277, 2007.

[13] C. Cai, R. Goebel, and A. R. Teel, "Smooth Lyapunov functions for hybrid systems Part II:(pre) asymptotically stable compact sets," IEEE Transactions on Automatic Control, vol. 53, no. 3, pp. 734-748, 2008.

[14] G. G. Yin and C. Zhu, Hybrid switching diffusions: properties and applications. Springer, 2009, vol. 63.

[15] S. P. Meyn and R. L. Tweedie, Markov chains and stochastic stability. Cambridge University Press, 2009.

[16] A. R. Teel, A. Subbaraman, and A. Sferlazza, "Stability analysis for stochastic hybrid systems: a survey," Automatica, vol. 50, no. 10, pp. 2435-2456, 2014.

[17] S. Venkateswaran, J. T. Isaacs, K. Fregene, R. Ratmansky, B. M. Sadler, J. P. Hespanha, and U. Madhow, "RF source-seeking by a micro aerial vehicle using rotation-based angle of arrival estimates," in Proceedings of the American Control Conference. IEEE, 2013, pp. 2581-2587.

[18] J. T. Isaacs, C. Magee, A. Subbaraman, F. Quitin, K. Fregene, A. R. Teel, U. Madhow, and J. P. Hespanha, "GPS-optimal micro air vehicle navigation in degraded environments," in Proceedings of the American Control Conference. IEEE, 2014, pp. 2581-2587.

[19] J. C. Martin and L. George, "Continuous state feedback guaranteeing uniform ultimate boundedness for uncertain dynamic systems," IEEE Transactions on Automatic Control, vol. 26, no. 5, p. 1139, 1981. 
[20] H. Lin and P. J. Antsaklis, "Uniformly ultimate boundedness control for uncertain switched linear systems," ISIS Technical Report, ISIS2003-004, University of Notre Dame, 2003.

[21] W. H. Sandholm, Population games and evolutionary dynamics. MIT press, 2010.

[22] E. Akin, The general topology of dynamical systems. American Mathematical Soc., 2010, vol. 1.

[23] A. Subbaraman and A. R. Teel, "A converse Lyapunov theorem for strong global recurrence," Automatica, vol. 49, no. 10, pp. 2963-2974, 2013.

[24] J. B. Pomet and L. Praly, "A result on robust boundedness,” Systems \& Control Letters, vol. 10, no. 2, pp. 83-92, 1988.

[25] R. Goebel, R. G. Sanfelice, and A. R. Teel, Hybrid dynamical systems: modeling, stability, and robustness. Princeton University Press, 2012.

[26] J. Poveda, A. Teel, and D. Nesic, "Flexible nash seeking using stochastic difference inclusions," in Proceedings of the American Control Conference, 2015.

[27] A. R. Teel, "On sequential compactness of solutions for a class of stochastic hybrid systems," in Proceedings of the American Control Conference, 2014, pp. 4512-4517.

[28] _ - "Lyapunov conditions certifying stability and recurrence for a class of stochastic hybrid systems," Annual Reviews in Control, vol. 37, no. 1 , pp. $1-24,2013$.

[29] R. Goebel and A. R. Teel, "Solutions to hybrid inclusions via set and graphical convergence with stability theory applications," Automatica, vol. 42, no. 4, pp. 573-587, 2006.

[30] A. Subbaraman and A. R. Teel, "A Krasovskii-Lasalle function based recurrence principle for a class of stochastic hybrid systems," in Proceedings of the 53rd IEEE Conference on Decision and Control, 2014, pp. 2310-2315.

[31] A. R. Teel, J. Hespanha, and A. Subbaraman, "A converse Lyapunov theorem and robustness for asymptotic stability in probability," IEEE Transactions on Automatic Control, pp. 2426-2441, 2014. 